\title{
Hospitalizations due to falls in Jewish and Arab children in northern Israel
}

\author{
Itai Shavit · Gad Bar-Joseph · Naim Shehadeh • \\ David Faraggi · Vardit Jan · Moshe Revach
}

Published online: 18 June 2009

(C) Springer Science+Business Media B.V. 2009

Erratum to: Eur J Epidemiol (2000) 16:47-52

DOI 10.1023/A:1007683020026

Due to a technical error, the names of the authors were inverted in the article $\mathrm{xml}$.

The online version of the original article can be found under doi: 10.1023/A:1007683020026.

I. Shavit $(\bowtie) \cdot$ N. Shehadeh $\cdot$ M. Revach Department of Pediatrics, Rambam Medical Center, Bruce Rappoport Faculty of Medicine, The Technion-Israel Institute of Technology, Haifa, Israel

e-mail: i_shavit@rambam.health.gov.il

G. Bar-Joseph

Pediatric Intensive Care Unit, Rambam Medical Center, Bruce Rappoport Faculty of Medicine, The Technion-Israel Institute of Technology, Haifa, Israel

D. Faraggi · V. Jan

Statistical Department of Haifa University, Haifa, Israel 